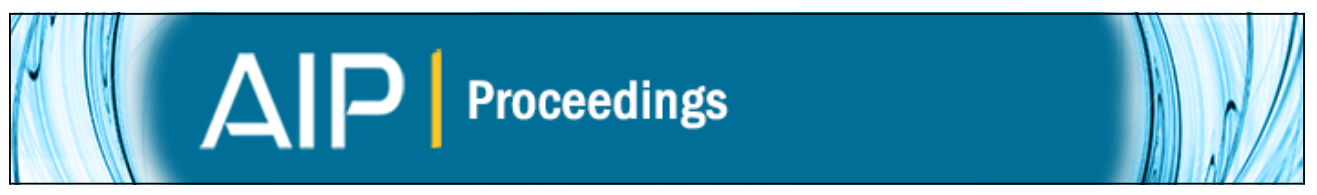

\title{
Vortices and chaos in the quantum fluid
}

D. A. Wisniacki, E. R. Pujals, and F. Borondo

Citation: AIP Conference Proceedings 905, 123 (2007); doi: 10.1063/1.2736998

View online: http://dx.doi.org/10.1063/1.2736998

View Table of Contents: http://scitation.aip.org/content/aip/proceeding/aipcp/905?ver=pdfcov

Published by the AIP Publishing

\section{Articles you may be interested in}

Decay of the vortex tangle at zero temperature and quasiclassical turbulence Low Temp. Phys. 39, 812 (2013); 10.1063/1.4821804

Semiclassical approach to model quantum fluids using the statistical associating fluid theory for systems with potentials of variable range

J. Chem. Phys. 136, 184506 (2012); 10.1063/1.4712299

Stability of giant vortices in quantum liquids

Chaos 14, 875 (2004); 10.1063/1.1785892

Quantum dynamics in simple fluids

J. Chem. Phys. 120, 6621 (2004); 10.1063/1.1645783

Quantum Chaos: An Introduction

Am. J. Phys. 68, 777 (2000); 10.1119/1.19544 


\title{
Vortices and chaos in the quantum fluid
}

\author{
D. A. Wisniacki*, E. R. Pujals ${ }^{\dagger}$ and F. Borondo** \\ *Departamento de Física "J.J. Giambiagi", FCEN, UBA, Pabellón 1, Ciudad Universitaria, 1428 \\ Buenos Aires, Argentina. \\ ${ }^{\dagger} I M P A-O S$, Dona Castorina 110, 22460-320, Rio de Janeiro, Brasil. \\ ${ }^{* *}$ Dep. de Química C-LX, Universidad Autónoma de Madrid, Cantoblanco, 28049 Madrid (Spain).
}

\begin{abstract}
The motion of a single vortex originates chaos in the quantum fluid defined in Bohm's interpretation of quantum mechanics. Here we analize this situation in a very simple case: one single vortex in a rectangular billiard.
\end{abstract}

Keywords: Quantum mechanics; Foundations of quantum mechanics; Measurement theory PACS: $03.65 . \mathrm{w}-, 03.65 . \mathrm{Ta}$

In the 1950's the physicist David Bohm presented a controversial formulation of quantum mechanics $(\mathrm{dBB})$ [1] with the purpose of solving some of the fundamental interpretational difficulties existing in the standard version. This theory is based on quantum trajectories (QT) "piloted" by the de Broglie's wave, and constitutes a true theory of quantum motion [2]. On the practical side, dBB has made possible very nice interpretations of physical phenomena such as diffraction [3], or tunneling [4], and also systematic quantum dynamical calculations in systems with many degrees of freedom [5]. Recently, it has also been used to attack other fundamental problems in physics (than those for which it was originally developed). Namely, Valentini and Westman [6] and Dürr et al. [7] made some start to remove the status of postulate to Born's probability rule: $\rho=|\psi|^{2}$. In these works, probabilities have a dynamical origin, similar to thermal probabilities in ordinary statistical mechanics, and it was shown [6] that the standard distribution is obtained as the time evolution towards the equilibrium of initial non-equilibrium states, $\rho \neq|\psi|^{2}$, this taking place with a (exponential) decrease in the associated coarse-grained $H$-function.

Underlying the above ideas is the assumption that it exists an effective chaotic dynamics in the QTs, something that has been frequently taken for granted [8], and/or used in a poorly justified way to make claims about the important topic of "quantum chaos". The main difficulty with this kind of approaches is the fact that trajectories in the de Broglie-Bohm (dBB) theory are non-local, and the flux is more similar to a hydrodynamical flow. Taking this into account, the authors have recently shown how the vortices of the pilot wave function are at heart of complexity of QT in dBB theory, by proving [9] that the motion of a single vortex is enough to create chaos in QT (using an isotropic 2D harmonic oscillator for a numerical illustration), being this a robust effect (numerically illustrated in a rectangular billiard) [10].

In this paper, we will consider the problem of a single vortex in a 2D square billiard, and give indications on how the study can be extended progressively.

In the $\mathrm{dBB}$ theory the state of the system is described by a wave function, $\psi(\mathbf{r}, t)=$

CP905, Frontiers of Fundamental Physics (FFP8), Eighth Intemational Symposium edited by B. G. Sidharth, A. Alfonso-Faus, and M. J. Fullana

(C) 2007 American Institute of Physics 978-0-7354-0412-0/07/\$23.00 
$R(\mathbf{r}, t) \mathrm{e}^{i S(\mathbf{r}, t)}$ ( $h=1$ throughout the paper), and the position of the particles, $\mathbf{r}$ [7]. The dynamical evolution of this two quantities (for spinless particles) is given by the timedependent Schrödinger equation and the guidance equation, respectively:

$$
\mathbf{v}=\dot{\mathbf{r}}=\frac{\nabla S}{m}=\frac{\mathbf{i}}{2 m} \frac{\psi \nabla \psi^{*}-\psi^{*} \nabla \psi}{|\psi|^{2}}
$$

where $m$ is the mass of the particle. QTs can be obtained by numerical integration of this equation. The velocity field (1), guiding these trajectories, presents singularities (at points where the wave functions vanishes) giving rise to vortices in quantum fluid. As a model we use a particle of mass $m=1 / 2$ enclosed in a 2D squared billiard with unit side length. The corresponding classical dynamics is totally regular, so that any indication of the existence of chaos is solely due to quantum effects. The corresponding eigenfunctions are: $\phi_{n_{x}, n_{y}}(x, y)=2 \sin \left(n_{x} \pi x\right) \sin \left(n_{y} \pi y\right)$, with wave numbers: $k=E_{n_{x}, n_{y}}^{1 / 2}=$ $\pi\left(n_{x}^{2}+n_{y}^{2}\right)^{1 / 2}$. The initial pilot wave is constructed as a linear combination of the first three eigenstates in the following way:

$$
\psi(x, y)=a \phi_{1,1}+b e^{i \alpha_{1}} \phi_{1,2}+c e^{i \alpha_{2}} \phi_{2,1}, \quad a, b, c, \alpha_{1}, \alpha_{2} \in \mathbb{R},
$$

with $a^{2}+b^{2}+c^{2}=1$ (normalization condition). Phases are chosen as: $\alpha_{1}=0, \alpha_{2}=\pi / 2$.

As can be easily demonstrated, this state generates a time-dependent velocity field with only one vortex, moving according to equation:

$$
\left(x_{v}(t), y_{v}(t)\right)=\frac{1}{\pi}\left(\arccos \frac{a \sin \left(\alpha_{2}-\omega\right) t}{2 b \sin \left(\alpha_{1}-\alpha_{2}\right)}, \arccos \frac{-a \sin \left(\alpha_{2}-\omega\right) t}{2 c \sin \left(\alpha_{1}-\alpha_{2}\right)}\right),
$$

with $\omega=E_{2,1}-E_{1,1}$. The corresponding vortex trajectories for different values of the parameters are shown in Fig. 1(a). As can be seen, they are approximately circular for the combination of lower values of $a$ and larger $b=c$, and distort to a more squared shape as the opposite happens. Due to the periodic character of the vortex motion, QTs (moving under the influence of the corresponding velocity field) can be best monitored in phase space by computing a "stroboscopic" surface of section (SOS) at times multiple of the fundamental frequency: $t=2 /(3 \pi)$. Let us recall that SOS, first described by Poincaré, are a fundamental tool in nonlinear science [11], so that much understanding of a dynamical system like our can be expected from their consideration. The corresponding results, for the same set of parameters used before, are shown in Fig. 1(b). In it, a transition to chaos takes place from regularity at $a / b=0$ to chaos when $a / b>0$, as a result of a pitchfork bifurcation. More interesting, the SOS shows all characteristics exhibited by ordinary generic autonomous systems. Namely, the portion or chaotic motion, or destroyed tori grows with the perturbation parameter $a / b$, giving the velocity of the vortex, and characteristics chains of islands are apparent for $a / b>0$. This is not surprising, since according to Ref. [9] the dynamics of the system in the vortex vicinity corresponds to that of a non-autonomous system of $1.5 \mathrm{D}$.

The next natural step in our research is to consider situations in which the number of vortices is progressively incremented. For these cases, interaction among vortices will exist, with the possibility of pair destruction maintaining the overall vorticity. The 


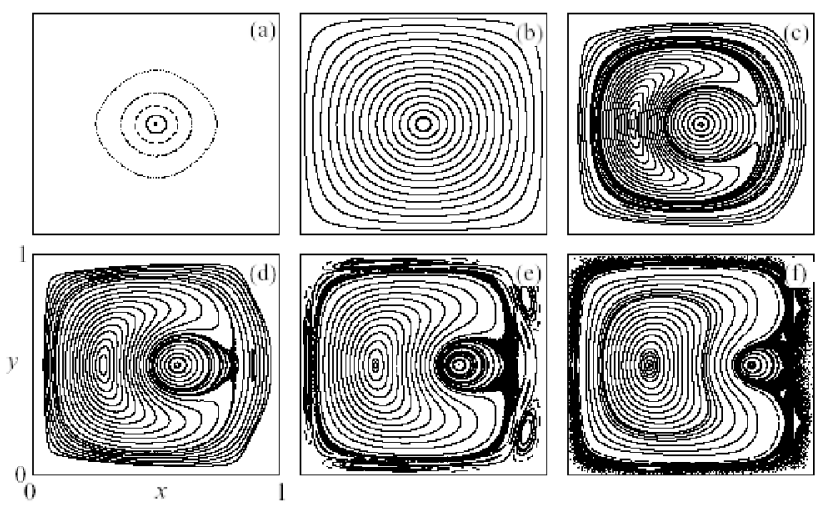

FIGURE 1. (a) Trajectories for the vortex generated by wave function (2) for $a=0, b=c=$ 0.707106781 (central point), $a=0.175, b=c=0.696195016$ (full line), $a=0.35, b=c=0.662382065$ (dashed), $a=0.525, b=c=0.601820156$ (dot-dashed), and $a=0.7, b=c=0.504975247$ (dotted).

(b)-(f) Composite stroboscopic Poincaré surfaces of section for QT's with the same conditions as in (a).

limit of many vortices originated by pilot wave functions of increasing complexity has been already numerically explored from a statistical point of view [10]. Now, it seems interesting to examine in detail the case of two vortices, which can be done with a generalization of the same technique used here, and this will be the subject of a future publication. The main idea is to transform the original non-autonomous flux into a dynamical system of $n+1 / 2$ dimensions, being $n$ the number of fundamental frequencies existing in the pilot wave function, and examine the corresponding phase space with surrogates of the associated Poincaré surface of section.

\section{ACKNOWLEDGMENTS}

This work was supported by CONICET and UBACYT (X248) (Argentina), MEC-Spain (contracts MTM2006-15533 and CSD2006-00032), AECI-Spain (A/4574/05).

\section{REFERENCES}

1. D. Bohm, Phys. Rev. 85, 166 (1952); ibid. 85, 194 (1952).

2. P. R. Holland, The Quantum Theory of Motion, Cambridge U. Press, Cambridge, 1993.

3. C. Philippidis, C. Dewdney, and B. J. Hiley, Nuovo Cimento 52B, 15 (1979).

4. C. L. Lopreore and R. E. Wyatt, Phys. Rev. Lett., 82, 5190 (1999).

5. R. E. Wyatt, Quantum Dynamics with Trajectories: Introduction to Quantum Hydrodynamics, Springer, New York, 2005.

6. A. Valentini and H. Westman, Proc. R. Soc. London, Ser. A 461, 253 (2005).

7. D. Dürr, S. Goldstein, and N. Zanghi, J. Stat. Phys. 67, 843 (1992).

8. H. Frisk, Phys. Lett. A 227, 139 (1997).

9. D. A. Wisniacki and E. R. Pujals, Europhys. Lett. 71, 159 (2005).

10. D. A. Wisniacki, E. R. Pujals and F. Borondo, Europhys. Lett. 73, 671 (2006).

11. M. A. Lieberman and A. J. Lichtenberg, Regular and Chaotic Dynamics, Springer, New York, 1992. 